RECyT

Año 23 / № 35 / 2021 / 14-21

\title{
Análisis sensorial de mate frío (tereré) de jugo artificial sabor naranja mediante estadística de supervivencia
}

\section{Sensory analysis of cold mate (tereré) with artificial orange juice using survival statistics}

\author{
Emiliano R. Neis*, Mónica M. Covinich, Griselda P. Scipioni \\ Facultad de Ciencias Exactas, Químicas y Naturales (FCEQyN), Universidad Nacional de \\ Misiones (UNaM). Félix de Azara 1552, Posadas, Misiones, Argentina. \\ *E-mail: emilianoneis@fceqyn.unam.edu.ar \\ Recibido: 11/08/2020; Aprobado 02/02/2021
}

\section{Resumen}

\begin{abstract}
Se realizó un ensayo de aceptabilidad sensorial para determinar la concentración óptima de jugo para preparar mate frío o tereré y un análisis descriptivo para estudiar la variación de la intensidad de cuatro descriptores seleccionados durante el consumo del tereré. Los resultados del análisis descriptivo fueron correlacionados con los valores de sólidos solubles obtenidos mediante la simulación del consumo del mate frío. Para la determinación de la concentración óptima de jugo se realizó un ensayo de aceptabilidad con consumidores, y los datos fueron analizados con herramientas de estadística de supervivencia. La concentración óptima de jugo fue de 6,38 \pm 0,62 $\mathrm{g} / \mathrm{L}$, que corresponde a un $22,87 \%$ de consumidores que rechazan la muestra. El ensayo descriptivo indicó que en los primeros tererés predomina el sabor del jugo y que posteriormente el sabor a yerba mate se intensifica gradualmente. Los coeficientes de correlación lineal entre los puntajes asignados a los descriptores sensoriales y las concentraciones de sólidos solubles de la simulación del consumo del mate sugieren que deben realizarse otros análisis representativos de la muestra a fin de establecer correlaciones más significativas.
\end{abstract}

Palabras clave: Mate frío; Yerba mate; Jugo artificial; Análisis sensorial; Estadística de supervivencia.

\section{Abstract}

A sensory acceptance test to determine the optimum concentration of artificial orange juice to prepare cold mate or tereré was performed, and a descriptive analysis to study the variation of four selected descriptors during tereré consumption was done. The results of the descriptive analysis were correlated with soluble solids values of a simulated cold mate consumption. Optimum juice concentration was determined by an acceptability test, and data were analyzed with survival statistics tools. The optimum concentration was $6.38 \pm 0.62 \mathrm{~g} / \mathrm{L}$, which matches to $22.87 \%$ of consumers rejecting the sample. The descriptive test indicated that, in the first tereré, the juice descriptors prevailed, and the yerba mate descriptors gradually intensified with successive tererés. Linear correlation coefficients found between sensory descriptors scores and soluble solids concentrations in the simulated consumption suggest that other representative determinations should be done to find more significant correlations.

Keywords: Cold mate; Yerba mate; Artificial juice; Sensory analysis; Survival statistics.

\section{Introducción}

La Yerba Mate (Ilex paraguariensis Saint Hilaire) es una planta cuyo uso tradicional es la preparación de infusiones, de amplio consumo en Argentina, Paraguay, Uruguay y el sur de Brasil [1]. El consumo interno de yerba mate en la Argentina durante el año 2019 fue de más de 277 millones de kilogramos [2]. El procesamiento industrial de este cultivo incluye etapas de cosecha, zapecado, secado, molienda y estacionamiento. En los países productores se emplean distintas variaciones del proceso de producción, lo que resulta en productos con propiedades organolépticas diferenciadas [3]-[5]. El consumo de yerba mate ha sido asociado con varios efectos benéficos sobre la salud, debido a sus propiedades antioxidantes, antiinflamatorias, antiobesidad y cardioprotectoras; lo que ha sido atribuido a la presencia de metabolitos secundarios como metilxantinas, polifenoles y saponinas [6], [7].

La yerba mate se consume como mate caliente tradicional, infusión en saquitos o mate cocido, y mate frío o tereré. El mate caliente se consume colocando yerba mate en un recipiente, y luego agregando alícuotas de agua a $70-85^{\circ} \mathrm{C}$. Cada alícuota es vertida sobre la misma yerba mate y luego bebida con una bombilla, constituyendo una 
cebada. El mate frío o tereré se consume de la misma manera, utilizando agua fría $\left(4-8^{\circ} \mathrm{C}\right)$. Finalmente, el mate cocido se prepara de forma similar al té [8], [9]. En el mercado existen yerbas con diferentes proporciones de hojas y palos, agregado de endulzantes, saborizantes y hierbas [10], [11].

Si bien el tereré se consume tradicionalmente con agua fría, también puede ser preparado utilizando jugos [12]. En la provincia de Misiones se emplean jugos naturales de frutas cítricas, aguas saborizadas y polvos para preparar bebidas saborizadas. Una particularidad de estos jugos en polvo es que la concentración con la que se preparan depende de la preferencia del consumidor. Para estimar la concentración de jugo preferida por los consumidores para la preparación del tereré pueden utilizarse los métodos de análisis de supervivencia.

El análisis de supervivencia estudia el tiempo hasta que ocurre un evento específico, llamado tiempo de supervivencia, teniendo en cuenta la presencia de datos censurados [13]. El fenómeno de censura se relaciona con la incertidumbre en la determinación del tiempo de un evento que, en muchos casos, no puede ser observado exactamente. Los métodos de análisis de supervivencia han sido utilizado en estudios clínicos, en epidemiología, en sociología, entre otros [14]. En ciencia de alimentos, los métodos de análisis de supervivencia han sido empleados para determinar la vida útil de un alimento, donde el evento de interés es el tiempo de almacenamiento; aunque también han sido utilizados para determinar la concentración óptima de un ingrediente en un alimento [15]-[17].

Las infusiones de yerba mate poseen sabor amargo característico, atribuido al contenido de metilxantinas (principalmente cafeína), compuestos polifenólicos, saponinas y taninos [4], [18]. Sin embargo, en cualquiera de las formas de consumo de la yerba mate, las infusiones pueden ser preparadas con el agregado de azúcar y otros edulcorantes y/o saborizantes [8], [11]; modificando la percepción del sabor amargo de la infusión. Por otra parte, varios autores han utilizado métodos que simulan el consumo de infusiones de yerba mate, y han encontrado que la composición de las mismas varía con las distintas cebadas [11], [19], [20]. Es de esperar que la variación de la composición de la infusión tenga influencia en el sabor de las mismas.

El análisis sensorial descriptivo es realizado por un grupo pequeño de panelistas, que proporcionan puntuaciones para un conjunto de atributos seleccionados [21]. Permite obtener una descripción sensorial completa de un producto, y establecer qué atributos son determinantes en la aceptación de un alimento [8]. Al momento, los autores no tienen conocimiento de otros trabajos realizados en análisis sensorial de tereré. El objetivo de este trabajo fue determinar la concentración de polvo para preparar jugo artificial sabor naranja preferida por los consumidores para la preparación de tereré, y determinar la variación de la intensidad de descriptores seleccionados de la misma bebida en función del número de cebada.

\section{Materiales y Métodos}

\section{Determinación de la concentración óptima de jugo}

\section{Análisis sensorial}

Se realizó un ensayo de aceptabilidad con 32 consumidores habituales de tereré (varones y mujeres de entre 22 y 60 años). Los participantes fueron reclutados de la Facultad de Ciencias Exactas, Químicas y Naturales de la Universidad Nacional de Misiones, siendo considerados aquellos que consumían tereré por lo menos dos veces por semana en los meses de verano. Se empleó la modalidad home use test, que permite garantizar las condiciones habituales de consumo de un alimento y tiene en cuenta la influencia de los efectos de preparación y consumo del producto, obteniendo de este modo datos más realistas [22], [23]. Cada consumidor recibió una planilla con instrucciones y cuatro paquetes codificados con números aleatorios de tres cifras. Cada paquete contenía una muestra de jugo en polvo, una muestra de yerba mate y una planilla con información del ensayo.

Se utilizó polvo para preparar jugo artificial sabor naranja, comercializado en sobres de 8 g cada uno, destinados a preparar un litro de jugo según las instrucciones del fabricante. A partir de conversaciones previas con consumidores habituales del producto se pudo inferir que la mayoría de ellos consume el jugo utilizando diluciones menores. Se ensayaron un total de cuatro concentraciones de jugo: 4, 6, 8 y 10 g/L. Cada consumidor recibió cuatro sobres con cantidad suficiente de polvo para preparar 500 $\mathrm{mL}$ de jugo de cada concentración. Cada sobre se preparó fraccionando cantidad suficiente de polvo para preparar la concentración adecuada. Los consumidores debían disolver el contenido del sobre en $500 \mathrm{~mL}$ de agua fría sin usar hielo, para no modificar la concentración de jugo a ensayar. La yerba utilizada fue la comercializada como "yerba mate para tereré". Según el rótulo del envase, esta yerba es de molienda más gruesa y tiene un menor contenido de polvo que la yerba mate elaborada tradicional. La cantidad de yerba utilizada en cada ensayo fue de $35 \mathrm{~g}$, como se sugiere en la norma IRAM 20540-1 [24] para la determinación de los caracteres organolépticos de la yerba mate. Los consumidores recibieron la misma cantidad de yerba mate para todas las concentraciones de jugo ensayadas.

La planilla entregada a cada consumidor contenía instrucciones generales para realizar el ensayo y para preparar el tereré: debían preparar los cuatros tererés en el lapso de una semana, en días diferentes, usando en cada ensayo el jugo y la yerba contenidos en uno de los sobres codificados. Se indicó a cada consumidor que preparara el jugo, colocara la yerba en el vaso que habitualmente usa para to- 
mar tereré y que bebiera, como lo hace habitualmente, seis cebadas de tereré sin compartirlo con otras personas; y que luego completara la planilla, respondiendo las preguntas correspondientes al sobre utilizado. La presentación de las muestras fue monádica y el ordenamiento fue balanceado.

Con objeto de comparar dos métodos estadísticos para la determinación de la concentración óptima de jugo en el tereré, se formularon dos preguntas de aceptabilidad para cada concentración: (1) puntuar el tereré según su agrado en una escala de 0 (nada) a 10 (mucho), respondiendo a la pregunta “¿Cuánto le agradó el tereré que tomó?”; (2) indicar su percepción con respecto a la concentración del jugo en el tereré, respondiendo a la pregunta "Este jugo me resultó:" con una de las opciones "Muy débil", "Está bien" o "Muy fuerte".

\section{Análisis estadístico}

Para los datos de la pregunta (1) se utilizó un análisis de varianza (ANOVA), realizado con el programa STATGRAPHICS Centurion XV (Versión 15.2.06). Se aplicó el test de Fischer LSD para calcular las diferencias entre medias. El análisis estadístico de los datos de la pregunta (2) fue realizado mediante herramientas de estadísticas de supervivencia en el programa estadístico RStudio (Versión 1.1.423); usando el código provisto por Hough [25]. El nivel de confianza utilizado en los análisis fue del 95\%.

\section{Análisis de supervivencia}

En el análisis de supervivencia se trabaja con datos censurados. Si un consumidor acepta el tereré con una concentración de jugo del 6\% pero rechaza el tereré con una concentración de jugo del $8 \%$; la concentración exacta de rechazo puede ser cualquier valor entre 6 y $8 \%$. Esto se define como censura en un intervalo. Cuando un consumidor rechaza la muestra con la menor concentración (4\%), la concentración de rechazo es $<4 \%$, lo que se define como censura a la izquierda. Si el consumidor acepta todas las concentraciones, el rechazo podría ocurrir para una concentración $>10 \%$, y los datos están censurados a la derecha [25].

Para determinar la concentración óptima de jugo se utilizó el modelo propuesto por Garitta et al. [16]. Para cada concentración de jugo $c$ habrá 2 funciones de rechazo: $R_{d}(c)$, la probabilidad de que un consumidor (o proporción de consumidores) rechace un tereré con la concentración de jugo $c$ porque es "muy débil"; y $R_{f}(c)$, la probabilidad de que un consumidor (o proporción de consumidores) rechace un tereré con la concentración de jugo $c$ porque es "muy fuerte". Se define como $C$ a la variable aleatoria que representa la concentración óptima de jugo para un consumidor.

$$
R_{d}(c)=P(C>c)=1-F(c)
$$

$R_{f}(c)=P(C<c)=F(c)$

En el análisis de supervivencia se utilizan varios modelos para $F(c)$ : Weibull, normal, lognormal, logístico, loglogístico, entre otros. Los parámetros del modelo elegido se obtienen maximizando la función de verosimilitud, y pueden estimarse para los datos experimentales usando software especializado [15]. Una descripción detallada de los métodos de análisis de supervivencia pueden encontrarse en el trabajo de Hough [25]. Este autor indica que una manera de definir el modelo que mejor ajusta a los datos experimentales es comparar los valores de logverosimilitud de diferentes modelos: el modelo que da los menores valores es el que resulta más adecuado.

El modelo de Garitta et al. [16] determina la concentración óptima de un ingrediente en un alimento como la concentración correspondiente al mínimo de la curva que surge de sumar las dos curvas de rechazo de los dos eventos de interés (de "muy débil" a “está bien", y de "está bien" a "muy fuerte"). Los intervalos de confianza de la concentración óptima se calculan como:

\section{Concentración óptima $\pm z_{1-\frac{\alpha}{2}} \cdot$ se promedio}

Donde $z$ es el parámetro de la distribución normal estándar, $\alpha$ es el nivel de confianza empleado y se promedio es el error estándar promedio, que se calcula como:

se promedio $=\frac{1}{2} \sqrt{s e_{1}^{2}+s e_{2}^{2}}$

Donde $s e_{1}$ y $s e_{2}$ son los errores estándar del óptimo calculado a partir de la curva de rechazo por "muy débil" y la curva de rechazo por "muy fuerte", respectivamente.

\section{Determinación de la variación de la intensidad de los descriptores con el número de cebada}

\section{Análisis sensorial}

Se realizó un análisis descriptivo con un panel sensorial de 10 evaluadores de la Facultad de Ciencias Exactas, Químicas y Naturales de la Universidad Nacional de Misiones, quienes son participantes habituales en ensayos sensoriales de yerba mate. Los evaluadores fueron instruidos en la mecánica de la prueba, y los descriptores seleccionados para ser medidos fueron "sabor a yerba mate", "sabor a naranja", "sabor dulce" y "sabor amargo", utilizando una escala de 0 (nada) a 10 (mucho).

El ensayo descriptivo fue realizado por duplicado en dos sesiones. En cada sesión, los panelistas recibieron un termo con jugo de naranja a $5^{\circ} \mathrm{C}$, un vaso de vidrio con yerba mate para tereré y una bombilla metálica. La 
concentración de jugo utilizada fue la determinada como óptima en el ensayo de aceptabilidad y se empleó la misma cantidad de yerba mate para tereré utilizada en dicho ensayo (35 g). Además, los evaluadores recibieron una planilla con espacios para registrar la intensidad de los descriptores para cada cebada por separado.

Con el objetivo de estandarizar el volumen de jugo utilizado en cada cebada, se realizaron ensayos previos para estimar la cantidad de líquido utilizada en el consumo habitual del tereré. En la primera cebada fueron necesarios $100 \mathrm{~mL}$ de jugo para humedecer toda la yerba, y en las cebadas siguientes se utilizaron $40 \mathrm{~mL}$ de jugo. Ambos volúmenes fueron marcados en vasos de plástico proporcionados a los panelistas. Se indicó a los evaluadores la manera de preparar el tereré: colocando la yerba en forma horizontal en el vaso e introduciendo la bombilla a $45^{\circ}$. En la primera cebada utilizaron $100 \mathrm{~mL}$ de jugo, y lo vertieron sobre la yerba en forma homogénea para humedecerla; mientras que en las cebadas posteriores usaron $40 \mathrm{~mL}$ de jugo en cada una. Los panelistas tomaron un total de seis cebadas de tereré, registrando respecto a cada una de ellas su percepción de los descriptores seleccionados.

\section{Análisis estadístico}

El análisis estadístico de los datos fue realizado con el programa STATGRAPHICS Centurion XV (v15.2.06). Los datos fueron analizados mediante un análisis de varianza (ANOVA) y se aplicó el test de Fischer LSD para calcular las diferencias entre medias. Las diferencias fueron consideradas significativas para $\mathrm{p}<0,05$.

\section{Simulación del consumo del mate frío}

Para determinar el contenido de sólidos solubles en cada cebada, se simuló el consumo de tereré en la forma realizada por los evaluadores en el ensayo descriptivo. Para ello se utilizó un sistema de extracción basado en otras investigaciones [9], [11], [20]. Se colocaron $35 \mathrm{~g}$ de yerba mate para tereré en un vaso de precipitado. Dentro del vaso se colocó una bombilla conectada a un matraz kitasato mediante un tubo plástico. El kitasato a su vez fue conectado a una trampa de vacío para realizar la succión. El líquido utilizado para la extracción fue el jugo en la concentración usada en el ensayo descriptivo y a la misma temperatura $\left(5^{\circ} \mathrm{C}\right)$. En cada cebada se usaron los mismos volúmenes usados en dicho ensayo $(100 \mathrm{~mL}$ en la primera cebada y $40 \mathrm{~mL}$ en las cebadas siguientes). La extracción fue realizada vertiendo el volumen correspondiente sobre la yerba mate, dejando reposar $30 \mathrm{~s}$, y aplicando vacío por otros $30 \mathrm{~s}$ para la extracción. El procedimiento se repitió por un total de seis cebadas. El volumen recuperado en cada cebada fue medido y guardado por separado. Los ensayos se realizaron por triplicado.

Los sólidos solubles presentes en el extracto fueron determinados por el método de pérdida de masa, colocando el extracto en una estufa a $100 \pm 2^{\circ} \mathrm{C}$ hasta peso constante. Con la misma técnica, se determinaron también los sólidos solubles del jugo utilizado para la extracción. Los datos obtenidos para cada cebada fueron correlacionados con los valores promedio de los descriptores evaluados en el ensayo anterior utilizando el programa STATGRAPHICS Centurion XV (Versión 15.2.06).

\section{Resultados y discusión}

\section{Concentración óptima de jugo para preparar tereré}

\section{Ensayo de aceptabilidad}

El análisis de varianza realizado para las respuestas de la pregunta (1) no resultó estadísticamente significativo $(\mathrm{p}>0,05)$ para ninguno de los factores estudiados. Dado que el factor "concentración" no resultó significativo, no pudo determinarse una concentración óptima de jugo a través de esta pregunta. En la Figura 1 se muestran los puntajes promedio asignados en la pregunta (1) a cada una de las concentraciones ensayadas.

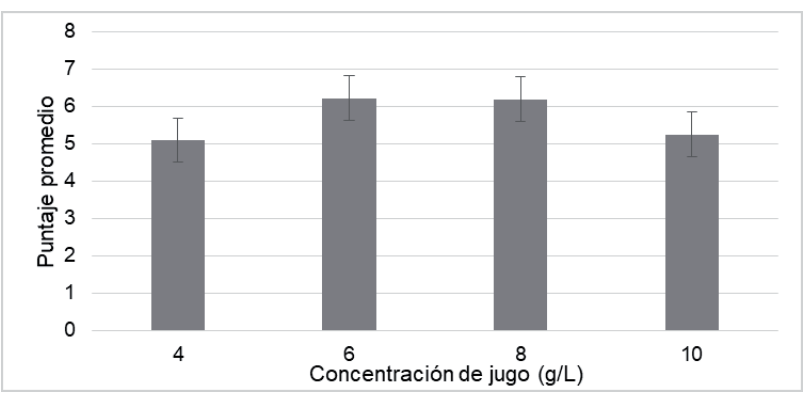

Figura 1: Puntajes promedio de los tererés preparados con distintas concentraciones de jugo

Con respecto al análisis de supervivencia, los modelos seleccionados para el ajuste de los datos fueron el modelo de Weibull para la curva de rechazo $R_{d}$ y el modelo lognormal para la curva de rechazo $R_{f}$ Para el modelo de Weibull, la función de rechazo está dada por:

$$
R_{d}(c)=1-F_{s e v}\left(\frac{\ln (c)-\mu_{d}}{\sigma_{d}}\right)
$$

Donde $F_{\text {sev }}(\cdot)$ es la función de la distribución del valor extremo, y $\mu_{d}$ y $\sigma_{d}$ son los parámetros del modelo [16]. Los resultados obtenidos fueron $\mu_{d}=1,605 \pm 0,102 ; \sigma_{d}=0,250$ $(0,172-0,362$; intervalos de confianza del $95 \%)$. En la Figura 2 se grafica el porcentaje de consumidores que rechazan el tereré por ser "muy débil", en función de la concentración de jugo. Se muestra que, por ejemplo, un tereré que sea preparado con jugo de concentración de $5 \mathrm{~g} / \mathrm{L}$, será rechazado por el $36,13 \%$ de los consumidores por ser muy débil. 


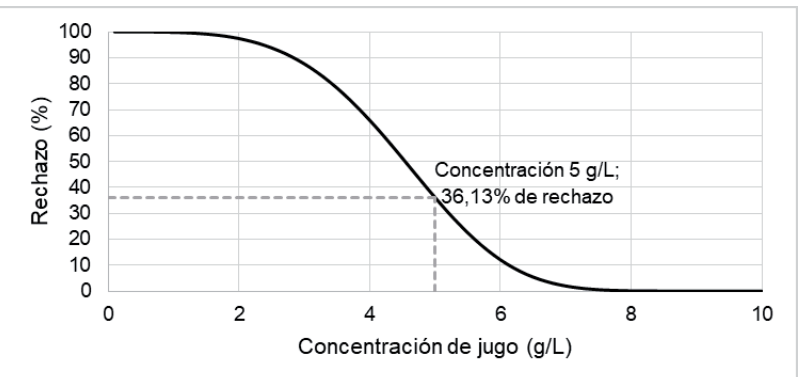

Figura 2: Porcentaje de consumidores que rechazan el tereré por ser "muy débil" en función de la concentración de jugo

Para la curva de rechazo $R_{f}$, correspondiente al rechazo del tereré por ser "muy fuerte", se seleccionó el modelo lognormal, dado por:

$R_{f}(c)=\Phi\left(\frac{\ln (c)-\mu_{f}}{\sigma_{f}}\right)$

Donde $\Phi(\cdot)$ es la función de la distribución normal estándar acumulativa, $\mathrm{y} \mu_{f} \mathrm{y} \sigma_{f}$ son los parámetros del modelo [15]. Para esta curva los parámetros encontrados fueron $\mu_{f}$ $=2,186 \pm 0,131$ y $\sigma_{f}=0,337(0,233-0,486$; intervalos de confianza del 95\%). En la Figura 3 se muestra la curva de rechazo del tereré por ser "muy fuerte".

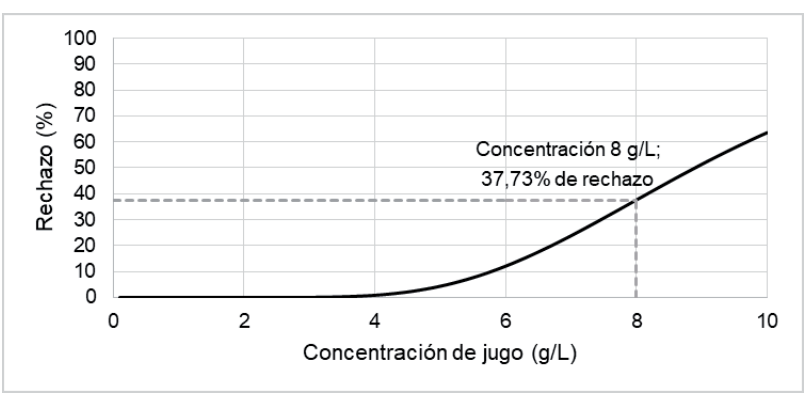

Figura 3: Porcentaje de consumidores que rechazan el tereré por ser "muy fuerte" en función de la concentración de jugo

En la Figura 4 se muestra la curva resultante de la suma de las dos curvas de rechazo de las Figuras 2 y 3. La concentración que corresponde al mínimo de la curva suma es la concentración óptima de jugo para preparar tereré, debido a que minimiza la suma de los porcentajes de rechazo. La concentración óptima resultó ser de 6,38 \pm $0,62 \mathrm{~g} / \mathrm{L}$, correspondiente a un $22,87 \%$ de rechazo. De ese porcentaje, un $6,72 \%$ rechaza el tereré preparado con jugo de esta concentración por ser muy débil, mientras que el $16,15 \%$ lo rechaza por ser muy fuerte. Considerando los resultados obtenidos puede afirmarse que efectivamente la mayor parte de la población de consumidores prefiere el tereré preparado con jugo de menor concentración a la recomendada por el fabricante $(8 \mathrm{~g} / \mathrm{L})$, teniendo esta concentración un rechazo del 37,73\%; por ser considerada muy fuerte (Figura 3).

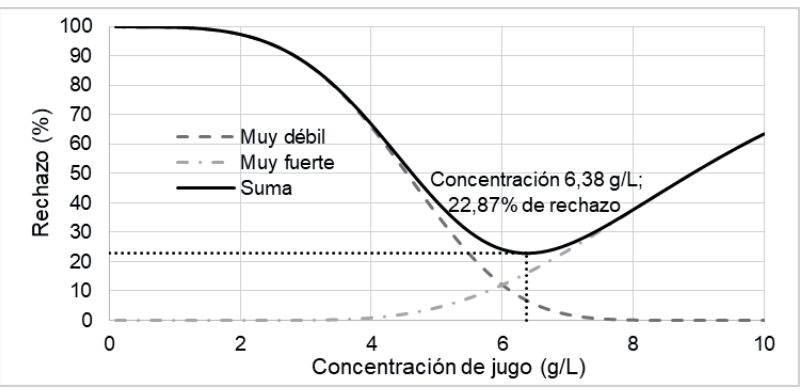

Figura 4: Suma de las curvas de rechazo y concentración óptima de jugo para tereré

\section{Variación de la intensidad de los descriptores con el número de cebada}

Los resultados de intensidad de los descriptores evaluados se muestran en la Figura 5. El número de cebada no mostró influencia significativa sobre la intensidad del sabor a yerba mate $(p>0,05)$. Las intensidades tanto del sabor a naranja como del sabor dulce disminuyeron con el número de cebada, observándose diferencias significativas a partir de la segunda y cuarta cebada, respectivamente. Por otro lado, se observó la tendencia opuesta en el descriptor "sabor amargo". En todos los casos, el efecto del evaluador fue significativo, indicando que los evaluadores emplearon zonas distintas de la escala.

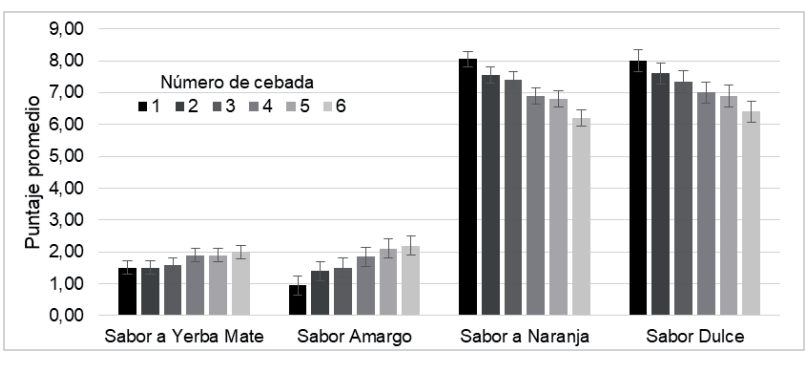

Figura 5: Variación de la intensidad de los descriptores seleccionados para tereré, en función del número de cebada.

Estos resultados indican que en las primeras cebadas de tereré predomina el sabor al jugo, asociado a los descriptores "sabor a naranja" y "sabor dulce"; mientras que en las cebadas siguientes se incrementa el sabor amargo, aportado por la yerba mate. Considerando una cinética típica de extracción, cabría esperar que en las primeras cebadas el sabor a yerba mate sea comparativamente más intenso que el sabor al jugo y en cebadas posteriores, cuando la yerba haya sido parcialmente agotada, se intensifique el sabor al jugo. Sin embargo, la tendencia encontrada en los ensayos es opuesta a la esperada y los evaluadores no pudieron identificar variaciones en el sabor a yerba mate con las cebadas. Esto sugiere que los descriptores asociados a la yerba mate fueron enmascarados por el sabor al jugo; lo que se evidencia en los puntajes comparativamente altos asignados a los descriptores característicos del jugo. En estudios posteriores podrían redefinirse los descriptores a 
ser ensayados, o bien repetir el ensayo usando concentraciones menores de jugo.

\section{Simulación del consumo del mate frío}

El contenido de sólidos solubles del jugo utilizado fue de 5,99 $\pm 0,31 \mathrm{~g} / \mathrm{L}$. En la Figura 6 se muestra el contenido de sólidos solubles presentes en cada cebada de tereré. El contenido de solubles de la primera cebada es el menor de las seis cebadas estudiadas, mientras que en la tercera cebada se alcanza un máximo en la concentración que luego disminuye gradualmente.

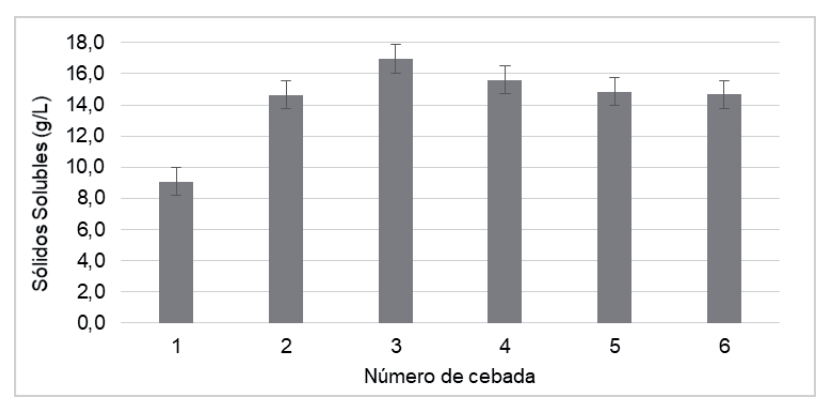

Figura 6: Sólidos solubles extraídos en función del número de cebada

Da Silvera et al. [19] estudiaron la extracción de compuestos fenólicos en el tereré, y encontraron que en las dos primeras cebadas hay un aumento en la concentración de estos compuestos, para alcanzar un máximo en la tercera cebada. En cebadas posteriores, observaron disminuciones graduales en el contenido de estos compuestos en los extractos. Meinhart et al. [20] encontraron tendencias similares para cafeína y teobromina en tereré, alcanzando un máximo de extracción en la segunda cebada. Ambos autores atribuyeron este comportamiento a que probablemente en la primera cebada la yerba no se encuentra totalmente humedecida. Los grupos de compuestos antes mencionados participan del sabor característico de la yerba mate [26], [27], por lo que esto podría explicar la tendencia observada con respecto a los descriptores asociados a la yerba mate: sabor a yerba mate y sabor amargo.

Los resultados de la simulación del consumo del tereré encontrados en este trabajo muestran una tendencia similar a la reportada por estos autores. En la extracción de un sólido con un líquido, luego de la separación del extracto, el sólido que contiene al soluto retiene cierta cantidad de solución adherida [28]. Parte del líquido agregado en las primeras cebadas conforma esta solución retenida, lo que resulta en que no toda la yerba sea humedecida y, consecuentemente, no participe en la extracción. Esto explicaría el hecho de que en la segunda y tercera cebada se logren extractos más concentrados que en la primera. A partir de la cuarta cebada se obtuvieron extractos de concentraciones gradualmente decrecientes, lo que sugiere que la yerba mate está siendo agotada en solutos.

Los coeficientes de correlación encontrados (Tabla
1) muestran correlaciones positivas muy bajas entre el contenido de sólidos solubles en cada cebada y los valores promedio de los descriptores encontrados en el ensayo descriptivo. Los coeficientes mostrados corresponden a correlaciones lineales. Se ensayaron otros tipos de correlación, encontrándose coeficientes igualmente bajos. La determinación de sólidos solubles no representa en forma adecuada la combinación compleja de caracteres organolépticos que componen el ensayo descriptivo; debido a que describe solamente un aspecto del extracto. A fin de encontrar correlaciones más significativas, en estudios posteriores podrían realizarse determinaciones de componentes más representativos de los descriptores estudiados, como cafeína para sabor amargo, edulcorantes para sabor dulce, entre otros.

Tabla 1: Coeficientes de correlación lineal entre los descriptores y la concentración de sólidos solubles

\begin{tabular}{|c|c|}
\hline Descriptor & Coeficiente de Correlación \\
\hline Sabor a Yerba mate & 0,3900 \\
\hline Sabor a Naranja & 0,5404 \\
\hline Sabor Dulce & 0,5706 \\
\hline Sabor Amargo & 0,6061 \\
\hline
\end{tabular}

\section{Conclusiones}

El análisis de estadística de supervivencia mostró ser más efectivo que la escala hedónica de 11 puntos para la determinación de la concentración óptima de jugo para preparar tereré. La concentración encontrada corresponde a un porcentaje de rechazo relativamente bajo, indicando que el producto es aceptado por los consumidores. Los resultados encontrados podrían emplearse como base para el desarrollo de jugos en polvo especialmente destinados para el consumo de tereré. El análisis descriptivo permitió proponer descriptores básicos para el tereré preparado con jugo, y estudiar la variación de los mismos con el número de cebada. Finalmente, el contenido de solidos solubles no pudo correlacionarse adecuadamente con las intensidades de los descriptores seleccionados.

\section{Agradecimientos}

Los autores agradecen la colaboración desinteresada del Dr. Guillermo Hough en la realización del trabajo.

\section{Referencias bibliográficas}

1. M. E. Schmalko, M. G. Acuña y G. P. Scipioni, "The Use of Maltodextrin Matrices to Control the Release of Minerals from Fortified Maté”, Int. J. Food Stud., vol. 1, no. 1, pp. 17-25, 2012.

2. Instituto Nacional de la Yerba Mate, "Informe del Sector Yerbatero. Diciembre 2019”, 2019. Disponible en: 
www.inym.org.ar/wp-content/uploads/2020/01/dic2019-estadisticas-inym-yerba-mate.pdf. Consultado el 28 de Julio de 2020.

3. E. L. Cardozo Junior y c. Morand, "Interest of mate (Ilex paraguariensis A. St.-Hil.) as a new natural functional food to preserve human cardiovascular health - A review", J. Funct. Foods, vol. 21, pp. 440-454, 2016.

4. S. A. Holowaty, S. A. Surkan, V. D. Trela, G. D. Byczko y M. E. Schmalko, "Variation of physicochemical and sensory properties during the aging of yerba mate", Int. J. Food Stud., vol. 3, no. 2, pp. 228-238, 2014.

5. S. Isolabella, L. Cogoi, P. López, C. Anesini, G. Ferraro y R. Filip, "Study of the bioactive compounds variation during yerba mate (Ilex paraguariensis) processing”, Food Chem., vol. 122, no. 3, pp. 695-699, 2010.

6. C. M. Pagliosa, M. A. Vieira, R. Podestá, M. Maraschin, A. L. Bertello Zeni, E. R. Amante, R. D. de M. C. Amboni, "Methylxanthines, phenolic composition, and antioxidant activity of bark from residues from mate tree harvesting (Ilex paraguariensis A. St. Hil.)”, Food Chem., vol. 122, no. 1, pp. 173-178, 2010.

7. M. Gómez-Juaristi, S. Martínez-López, B. Sarria, L. Bravo y R. Mateos, "Absorption and metabolism of yerba mate phenolic compounds in humans", Food Chem., vol. 240, pp. 10281038, 2018.

8. M. J. Santa Cruz, L. Garitta y G. Hough, "Sensory Descriptive Analysis of Yerba Mate (Ilex Paraguariensis Saint Hilaire), a South American Beverage”, Food Sci. Technol. Int., vol. 8, no. 1, pp. 25-31, 2002.

9. A. E. Thea, D. Ferreira, L. A. Brumovsky y M. E. Schmalko, "POlycyclic aromatic hydrocarbons (PAHs) in yerba maté (Ilex paraguariensis St. Hil) traditional infusions (mate and tereré)", Food Control, vol. 60, pp. 215220, 2016.

10. C. S. Bizzotto, A. D. Meinhart, A. C. P. Rybka, M. R. Sobrinho, S. B. Junior, C. A. Ballus y H. T. Godoy, "Quantification of phenolic compounds by capillary zone electrophoresis in extracts of four commercial types of mate herb before and after acid hydrolysis", Food Res. Int., vol. 48, no. 2, pp. 763-768, 2012.

11. G. P. Scipioni, D. J. Ferreyra, M. G. Acuña y M. E. Schmalko, "Rebaudioside A release from matrices used in a yerba maté infusion”, J. Food Eng., vol. 100, no. 4, pp. 627-633, 2010.

12. F. Cúneo y N. Schaab, "Hábitos de consumo de bebidas en adolescentes y su impacto en la dieta”, Diaeta, vol. 31, no. 142, pp. 34-41, 2013.

13. G. Hough, L. Garitta y R. Sánchez, "Determination of consumer acceptance limits to sensory defects using survival analysis", Food Qual. Prefer., vol. 15, no. 7-8, pp. 729-734, 2004.

14. Y. Jeong, Y. A. Jang, Y. H. Chang y Y. Lee, "Prediction of consumer acceptability of potato chips with various moisture contents using survival analysis", Food Sci. Biotechnol., vol. 22, no. 6, pp. 1621-1627, 2013.
15. E. A. Esmerino, J. A. Paixão, A. G. Cruz, L. Garitta, G. Hough y H. M. A. Bolini, "Survival analysis: A consumer-friendly method to estimate the optimum sucrose level in probiotic petit suisse”, J. Dairy Sci., vol. 98, no. 11, pp. 7544-7551, 2015.

16. L. v. Garitta, C. Serrat, G. E. Hough y A. v. Curia, "Determination of optimum concentrations of a food ingredient using survival analysis statistics", J. Food Sci., vol. 71, no. 7, pp. S526-S532, 2006.

17. M. Sosa, A. Flores, G. Hough, N. Apro, V. Ferreyra y M. M. Orbea, "Optimum level of salt in French-type bread. Influence of income status, salt level in daily bread consumption, and test location", J. Food Sci., vol. 73, no. 8, pp. S392-S397, 2008.

18. C. M. Pagliosa, S. M. Pereira, M. A. Vieira, L. A. Costa, E. Teixeira, R. D. de M. C. Amboni y E. R. Amante, "Bitterness in yerba mate (Ilex Paraguariensis) leaves”, J. Sens. Stud., vol. 24, no. 3, pp. 415-426, 2009.

19. T. F. F. da Silveira, A. D. Meinhart, T. C. L. de Souza, E. C. E. Cunha, M. R. de Moraes y H. T. Godoy, "Chlorogenic acids and flavonoid extraction during the preparation of yerba mate based beverages", Food Res. Int., vol. 102, pp. 348-354, 2017.

20. A. D. Meinhart, C. S. Bizzotto, C. A. Ballus, A. C. P. Rybka, M. R. Sobrinho, R. S. Cerro-Quintana, J. Teixeira-Filho, H. T. Godoy, "Methylxanthines and phenolics content extracted during the consumption of mate (IIex paraguariensis St. HiI) beverages", J. Agric. Food Chem., vol. 58, no. 4, pp. 2188-2193, 2010.

21. D. Valentin, S. Chollet, M. Lelièvre y H. Abdi, "Quick and dirty but still pretty good: A review of new descriptive methods in food science”, Int. J. Food Sci. Technol., vol. 47, no. 8, pp. 1563-1578, 2012.

22. E. de Hooge, M. Oostindjer, J. Aschemann-Witzel, A. Normann, S. M. Loose y V. L. Almli, “This apple is too ugly for me!: Consumer preferences for suboptimal food products in the supermarket and at home”, Food Qual. Prefer., vol. 56, pp. 80-92, 2017.

23. Boutrolle, D. Arranz, M. Rogeaux y J. Delarue, "Comparing central location test and home use test results: Application of a new criterion”, Food Qual. Prefer., vol. 16, no. 8, pp. 704-713, 2005.

24. Instituto Argentino de Racionalización de Materiales (IRAM), "Norma 20540-1: Yerba mate: Materiales y procedimientos a utilizar en la determinación de los caracteres organolépticos de la yerba mate, bajo forma de mate", 1997.

25. G. Hough, Sensory shelf life estimation of food products. Boca Raton, FL: CRC Press, 2010.

26. N. M. Streit, L. H. R. Hecktheuer, M. W. do Canto, C. A. Mallmann, L. Streck, T. V. Parodi, L. P. Canterle, "Relation among tasterelated compounds (phenolics and caffeine) and sensory profile of erva-mate (Ilex paraguariensis)", Food Chem., vol. 102, no. 3, pp. 560-564, 2007.

27. I. Lesschaeve y A. C. Noble, "Polyphenols: factors influen- 
cing their sensory properties and their effects on food and beverage preferences", Am. J. Clin. Nutr., vol. 81, no. 1, pp. 330S-335S, 2005.

28. A. Ibarz y G. v. Barbosa Cánovas, Operaciones unitarias en la ingeniería de alimentos. Madrid, España: Ediciones Mundi-Prensa, 2005. 